Original Article

\title{
The Effects of Tai Chi on the Renal and Cardiac Functions of Patients with Chronic Kidney and Cardiovascular Diseases
}

\author{
Zhi-Min Shi, MS ${ }^{1)}$, Hai-Ping Wen, MS ${ }^{1)}$, Fu-Rong Liu, MS ${ }^{2)}$, Chun-Xia YaO, PhD ${ }^{1)^{*}}$ \\ 1) Medical College of Hebei University of Engineering: Cong Tai Road 83, Handan 056002, Hebei \\ Province, China \\ 2) HanDan Central Hospital, China
}

\begin{abstract}
Purpose] To assess the effects of Tai Chi on the renal and cardiac functions of patients with chronic kidney disease (CKD) and cardiovascular disease (CVD). [Subjects and Methods] Twenty-one patients with CKD and CVD were randomly divided into control and exercise groups. The exercise group performed Tai Chi training for 30 minutes three to five times a week for 12 weeks, while the control group did not. All patients' renal and cardiac functions and blood lipid parameters were measured at baseline and after 12 weeks. [Results] The 12 weeks Tai Chi intervention improved the estimated glomerular filtration rate (eGFR), left ventricular ejection fraction (LVEF), and the high density lipoprotein (HDL) level, and decreased the serum creatintine (Scr) level, heart rate (HR), systolic blood pressure (SBP), diastolic blood pressure (DBP), and the total cholesterol (CH), triglyceride (TG) and low density lipoprotein (LDL) levels. The change in eGFR correlated negatively with the changes in CH, TG and LDL, and positively with the change in HDL. In addition, the change in SBP correlated positively with the changes in $\mathrm{CH}$, TG and LDL, and negatively with the change in HDL. [Conclusion] Tai Chi training might improve the renal and cardiac functions of CKD and CVD patients via improved regulation of lipid metabolism.

Key words: Tai Chi, Renal function, Lipid metabolism
\end{abstract}

(This article was submitted Feb. 26, 2014, and was accepted May 16, 2014)

\section{INTRODUCTION}

Chronic kidney disease (CKD), also known as chronic renal disease, is a gradual loss of kidney function over time. Many studies have demonstrated that CKD patients face increased risk of cardiovascular disease (CVD), which has a significant impact upon patient mortality ${ }^{1-3}$. Therefore, the prevention and treatment of CVD has become the key to the management of patients with CKD.

Patients with a range of CKD and CVD symptoms suffered from poor physical performance. Sietsema et al. found that reduced exercise capacity was associated with poorer survival among ambulatory patients with end-stage renal diseases ${ }^{4}$. Stack et al. also reported that physical exercise reduced by about $30 \%$ the risk of death of patients receiving hemodialysis ${ }^{5)}$. Moreover, some studies have shown that appropriate physical activity such as aerobic exercise can improve the renal and cardiac functions of individuals with $\mathrm{CKD}^{6-8)}$. Based on these observations, CKD patients are encouraged to exercise, particularly aerobic and resistance exercises $^{9,10)}$. Examples of aerobic exercise are brisk walk-

*Corresponding author. Chun-Xia Yao (E-mail: yaoyao20050208@163.com)

(C2014 The Society of Physical Therapy Science. Published by IPEC Inc. This is an open-access article distributed under the terms of the Creative Commons Attribution Non-Commercial No Derivatives (by-ncnd) License $<$ http://creativecommons.org/licenses/by-nc-nd/3.0/> ing, jogging, swimming, cycling, climbing stairs, and Tai Chi and et al. Tai Chi is a traditional Chinese exercise which has moderate intensity, and it has been found to benefit for healthy persons and patients with Parkinson's disease, heart failure, myocardial infarction, and hypertension, to improving the quality of life and cardiovascular function ${ }^{11-13)}$.

As a type of exercise for health and fitness, Tai Chi is widely practiced in many countries. However, the clinical effects and molecular mechanisms of Tai Chi in CKD patients have not been well studied. Therefore, it is important to further evaluate the effects of Tai Chi on CKD patients. In this study, we performed a clinical study and investigated whether Tai Chi can improve the renal and cardiac functions of patients with CKD and CVD.

\section{SUBJECTS AND METHODS}

This study was a 12 week randomized controlled trial which included 21 CKD patients with CVD. The patients were randomly divided into exercise $(n=11)$ and control $(\mathrm{n}=10)$ groups. The exercise group performed Tai Chi training for 30 minutes three to five times a week for 12 weeks, while the control group did not. Both groups received routine medical treatment for CVD and CKD. We tested all patients' renal and cardiac functions and blood lipid parameters at baseline and after 12 weeks.

The study protocol was carried out in accordance with the Declaration of Helsinki and was approved by the Medi- 
cal College's Ethics Committee of Hebei University of Engineering. Written informed consent was obtained from each patient.

A total of 21 patients with CKD and CVD (male/female: 14/7; mean age: 69.4 \pm 7.7 years; left ventricular ejection fraction $56.4 \pm 12.6 \%$ ) were recruited from Han Dan Central Hospital in China. The baseline characteristics of the study population are given in Table 1. Exclusion criteria were: left ventricular ejection fraction $\leq 30 \%$; proteinuria $\geq 1 \mathrm{~g} /$ day; nephritic syndrome; rapidly progressive golmerulonephritis; history of low pulmonary function; unable to exercise.

The Tai Chi intervention consisted of 10 minutes of warm-up exercises, 30 minutes of Tai Chi practice and 5 minutes of cool-down exercises. Ten core movements were chosen from classical Yang-style Tai Chi and taught by 1 or 2 experienced instructors. After four weeks of supervised training, patients were encouraged to continue their exercise program unsupervised at home at least three to five times per week for eight weeks. Home practice was tracked via self-report logs.

Blood urea nitrogen (BUN), serum creatintine (Scr), total cholesterol $(\mathrm{CH})$, triglyceride (TG), low density lipoprotein (LDL) and high density lipoprotein (HDL) were evaluated using standard laboratory methods on a Hitachi 912 Chemistry Analyzer (Roche Diagnostics, Germany). eGFR was estimated by using the following equation of the Modification of Diet in Renal Disease Study for Chinese: eGFR $\left(\mathrm{ml} \cdot \mathrm{min}^{-1} \cdot 1.73 \mathrm{~m}^{-2}\right)=186 \times[\mathrm{Scr}]^{-1.154} \times[\mathrm{age}]^{-0.203} \times[0.742$ (if female) $] \times[1.233 \text { (Chinese coefficient) }]^{14)}$.

BP was measured by an expert physician using a stethoscope and a sphygmomanometer via the auscultatory method while the patients were relaxed. The first and last Korotkoff sounds were recorded as systolic and diastolic blood pressure (SBP and DBP), respectively.

The echocardiographic examination was performed by two experienced specialists using a GE Vivid 7 or Vivid 9 ultrasound systems. The measurements included left ventricular diastolic diameter (LVDD), left ventricular systolic diameter (LVSD) and left ventricular ejection fraction (LVEF).

All data are expressed as mean \pm SEM. The two-way analysis of variance was performed to detect statistical differences. The Mann-Whitney U test and the Wilcoxon test were respectively performed to investigate the differences between groups, and between baseline and after 12 weeks intervention. Association of eGFR and blood lipid parameters was evaluated using Pearson's correlation coefficient. All statistical analyses were carried out using a commercially available statistical package SPSS version 12.0. Values of $\mathrm{p}<0.05$ were considered statistically significant.

\section{RESULTS}

No differences were observed between the control and the exercise groups with respect to renal function, blood lipid parameters, BP and cardiac function at baseline (Table 1). All of the 21 patients fully completed the study. Medical treatment was maintained constantly, and no clinical events were recorded during the study period.
Table 1. Baseline characteristics

\begin{tabular}{lccc}
\hline Characteristic & $\begin{array}{c}\text { Control } \\
(\mathrm{n}=10)\end{array}$ & $\begin{array}{c}\text { Tai Chi } \\
(\mathrm{n}=11)\end{array}$ & $\mathrm{p}$ \\
\hline Age (years) & $68.4 \pm 8.7$ & $69.5 \pm 6.9$ \\
Male sex & 6 & 9 \\
Left ventricular ejection & $56.3 \pm 13.9$ & $56.5 \pm 12.3$ \\
fraction (\%) & 4 & 7 \\
Beta-blocker & 0 & 1 \\
Angiotensin II antagonist & 5 & 6 \\
Angiotensin-coverting & 2 & 3 \\
enzyme inhibitor & 2 & 3 \\
Ca-antagonist & 5 & 6 \\
Nitrate & 6 & 9 \\
Myocardial infarction & 5 & 7 \\
Hypertension & 2 & 5 \\
Hyperlipidemia & 0 & 0 \\
Diabetes & & \\
Smoking & &
\end{tabular}

As shown in Table 2, 12 weeks of regular Tai Chi intervention significantly improved eGFR in the exercise group, but we did not find a change in the control group. There were significant differences in the changes of eGFR and Scr between the two groups. Although, the exercise had no significant effect on BUN in either group, the change of BUN in the exercise group showed a slight decline trend compared to the control group.

Compared with baseline, 12 weeks of Tai Chi exercise decreased $\mathrm{CH}$, TG and LDL levels and increased the HDL levels (Table 3); no differences were seen in control group. The changes in blood lipid parameters were markedly different between the control and the exercise groups.

Table 4 shows that SBP, DBP and HR reduced notably after 12 weeks of Tai Chi exercise; there was no variations were observed in the control group. The SBP, DBP and HR in the exercise group after 12 weeks of Tai Chi intervention were clearly lower than the values of the control group which did not perform Tai Chi exercise.

After 12 weeks of Tai Chi exercise, LVEF in the exercise group had increased by an average of 10\% compared to baseline in spite of there being no change in LVDD and LVSD (Table 4). Moreover, HR in the exercise group decreased, but we failed to find a significant change in the control group. The changes in HR and LVEF in the exercise group after 12 weeks of Tai Chi intervention had showed significant differences from those of in the control group.

In the exercise group, the change in eGFR negatively correlated with the changes in $\mathrm{CH}, \mathrm{TG}$ and LDL, and positively with the change in HDL (Table 5). Also, the change in SBP correlated positively with the changes in $\mathrm{CH}$, TG and LDL, and negatively with the change in HDL.

\section{DISCUSSION}

We evaluated the effects of Tai Chi training on the renal, and cardiac functions and blood lipid parameters of patients 
Table 2. Comparison of renal function between baseline and 12 weeks

\begin{tabular}{lrrrc}
\hline & \multicolumn{2}{c}{ Control group } & \multicolumn{2}{c}{ Exercise group } \\
\cline { 2 - 5 } & Baseline & 12 weeks & Baseline & 12 weeks \\
\hline BUN $(\mathrm{mg} / \mathrm{dl})$ & $25.1 \pm 3.2$ & $28.0 \pm 2.6$ & $25.6 \pm 2.4$ & $24.2 \pm 1.6$ \\
$\mathrm{Scr}(\mathrm{mg} / \mathrm{dl})$ & $1.3 \pm 0.2$ & $1.5 \pm 0.3$ & $1.3 \pm 0.3$ & $1.1 \pm 0.2^{\Delta}$ \\
$\mathrm{eGFR}\left(\mathrm{ml} \cdot \mathrm{min}^{-1} \cdot 1.73 \mathrm{~m}^{-2}\right)$ & $45 \pm 8.5$ & $43 \pm 7.8$ & $45 \pm 7.6$ & $51 \pm 6.5^{* \Delta}$ \\
\hline
\end{tabular}

${ }^{*} \mathrm{p}<0.05$ compared with the value at baseline, by the Wilcoxon's test. ${ }^{\Delta} \mathrm{p}<0.05$ compared with the value in control group, by the Mann-Whitney U test. BUN, blood urea nitrogen; eGFR, estimated glomerular filtration rate; Scr, serum creatinine

Table 3. Comparison of lipid parameters between baseline and 12 weeks

\begin{tabular}{lcccc}
\hline & \multicolumn{2}{c}{ Control group } & \multicolumn{2}{c}{ Exercise group } \\
\cline { 2 - 5 } & Baseline & 12 weeks & Baseline & 12 weeks \\
\hline $\mathrm{CH}(\mathrm{mmol} / \mathrm{L})$ & $5.63 \pm 0.77$ & $5.70 \pm 0.83$ & $5.60 \pm 0.81$ & $5.03 \pm 0.92^{* \Delta}$ \\
$\mathrm{TG}(\mathrm{mmol} / \mathrm{L})$ & $1.51 \pm 0.78$ & $1.54 \pm 0.68$ & $1.56 \pm 0.73$ & $1.41 \pm 0.55^{* \Delta}$ \\
$\mathrm{LDL}(\mathrm{mmol} / \mathrm{L})$ & $3.73 \pm 0.82$ & $3.78 \pm 0.56$ & $3.67 \pm 0.89$ & $3.36 \pm 0.44^{* \Delta}$ \\
$\mathrm{HDL}(\mathrm{mmol} / \mathrm{L})$ & $1.15 \pm 0.51$ & $1.11 \pm 0.60$ & $1.08 \pm 0.56$ & $1.18 \pm 0.37^{* \Delta}$ \\
\hline
\end{tabular}

${ }^{*} \mathrm{p}<0.05$ compared with the value at baseline, by the Wilcoxon's test. ${ }^{\mathrm{p}} \mathrm{p}<0.05$ compared with the value in control group, by the Mann-Whitney U test. $\mathrm{CH}$, total cholesterol; HDL, high density lipoprotein; LDL, low density lipoprotein; TG, triglyceride

Table 4. Comparison of cardiac function between baseline and 12 weeks

\begin{tabular}{lcccc}
\hline & \multicolumn{2}{c}{ Control group } & \multicolumn{2}{c}{ Exercise group } \\
\cline { 2 - 5 } & Baseline & 12 weeks & Baseline & 12 weeks \\
\hline SBP (mmHg) & $145 \pm 21$ & $149 \pm 19$ & $142 \pm 18$ & $129 \pm 16^{* \Delta}$ \\
DBP (mmHg) & $89 \pm 12$ & $92 \pm 11$ & $88 \pm 13$ & $80 \pm 10^{* \Delta}$ \\
HR (bpm) & $72 \pm 11$ & $73 \pm 12$ & $74 \pm 10$ & $65 \pm 8^{* \Delta}$ \\
LVEF (\%) & $56.3 \pm 13.9$ & $57.1 \pm 12.2$ & $56.5 \pm 12.3$ & $63.5 \pm 10.7^{* \Delta}$ \\
LVDD (mm) & $44.0 \pm 6.0$ & $43.9 \pm 6.9$ & $44.2 \pm 6.6$ & $43.8 \pm 4.7$ \\
LVSD (mm) & $31.9 \pm 5.7$ & $32.5 \pm 4.5$ & $32.4 \pm 4.4$ & $31.2 \pm 3.8$ \\
\hline
\end{tabular}

$* \mathrm{p}<0.05$ compared with the value at baseline, by the Wilcoxon's test. ${ }^{\Delta} \mathrm{p}<0.05$ compared with the value in control group, by the Mann-Whitney U test. DBP, diastolic blood pressure; HR, heart rate; LVDD, left ventricular diastolic diameter; LVEF, left ventricular ejection fraction; LVSD, left ventricular systolic diameter; SBP, systolic blood pressure

Table 5. Correlation of exercise group changes in eGFR, SBP and lipid parameters

\begin{tabular}{lrcrc}
\hline & \multicolumn{2}{c}{ eGFR } & \multicolumn{3}{c}{ SBP } \\
\cline { 2 - 5 } & $\mathrm{r}$ & $\mathrm{p}$ & $\mathrm{r}$ & $\mathrm{p}$ \\
\hline $\mathrm{CH}(\mathrm{mmol} / \mathrm{L})$ & -0.462 & 0.036 & 0.487 & 0.035 \\
TG $(\mathrm{mmol} / \mathrm{L})$ & -0.512 & 0.025 & 0.545 & 0.012 \\
LDL (mmol/L) & -0.536 & 0.018 & 0.529 & 0.021 \\
HDL (mmol/L) & 0.675 & 0.001 & -0.633 & 0.002 \\
\hline
\end{tabular}

$\mathrm{CH}$, total cholesterol; eGFR, estimated glomerular filtration rate; HDL, high density lipoprotein; LDL, low density lipoprotein; SBP, systolic blood pressure; TG, triglyceride

with CKD and CVD. The 12 weeks of Tai Chi training resulted in significant changes in eGFR, $\mathrm{CH}, \mathrm{TG}, \mathrm{LDL}, \mathrm{HDL}$, HR, SBP, DBP, and LVEF in the exercise group while no changes were observed in the control group. To the best of our knowledge, this is the first clinical study to showing the potential benefits of Tai Chi training in CKD and CVD patients.
The presence of high TG and low HDL, as is well documented in previous reports, accelerates the progression of $\mathrm{CKD}^{15-17)}$. Aerobic exercise usually decreases TG and increases HDL in patients with $\mathrm{CVD}^{18,19)}$. Moreover, several studies have indicated that the elevation of the HDL level could improve renal function ${ }^{20,21)}$. Our results are consistent with these previous reports, which suggest that regular 
Tai Chi training can improve the renal and cardiac functions through promotion of blood lipid metabolism, and especially HDL elevation. Consequently, Tai Chi appears to be an effective exercise for preventing the progression of CKD and CVD.

In spite of abundant evidence of the benefits of exercise for CKD and CVD patients ${ }^{4-8,17-19)}$, and the fact that exercise was first reported to improved abnormal metabolism in patients with CKD was first introduced 3 decades ago ${ }^{22)}$, therapeutic exercise for advanced CKD patients is still not widespread. What are the causes of the current situation? One possibility is that there is no specific exercise guideline for this group of patients yet. Another reason may be that the majority CKD and CVD patients fail to keep walking, jogging, swimming, cycling or climbing, because their condition makes them susceptible to fatigue rendering them to persist with such exercises. A third possibility is that some exercises require equipment which is unavailable for outpatients. Therefore, it is necessary to find a simple, easy, suitably available and effective way of exercise for patients with CKD and CVD. In view of the above requirements, Tai Chi is undoubtedly the best choice. Ours and others studies have demonstrated the broad benefits of Tai Chi for CKD and CVD patients ${ }^{23-25)}$.

Our study had several limitations. First, the present study was a small sample size pilot study, which only included 21 patients, and our results need to be confirmed in further larger trails. Second, the intervention period was only 12 weeks, and it is necessary to examine the long-term effects of Tai Chi training. Third, the lack of blinding with patients may have influenced our results. Furthermore, our study didn't take gender, social status or severity of the disease into account. In addition, further studies are also needed to explore the combination effect of Tai Chi with resistance exercise on CKD and CVD. However, since the aim of our study was to confirm the beneficial effects of Tai Chi training on the renal and cardiac functions of patients with CKD and CVD, and we believe it is a valuable study in that it has provided results which warrant.

Our results confirmed Tai Chi training might improve the renal and cardiac functions of patients with CKD and CVD through modification of blood lipid metabolism. Although further investigations are needed to uncover the mechanisms behind the improvements, Tai Chi may be an easy and effective clinical treatment strategy for preventing the progression of CKD and CVD, enhancing live quality of life, and increasing life span.

\section{REFERENCES}

1) Blaine J, Levi M: Chronic kidney disease: albuminuria or CKD stag as best marker of CVD in diabetes? Nat Rev Nephrol, 2012, 8: 376-377. [Medline] [CrossRef]

2) Schiffrin EL, Lipman ML, Mann JF: Chronic kidney disease: effects on the cardiovascular system. Circulation, 2007, 116: 85-97. [Medline] [CrossRef]

3) Shlipak MG, Fried LF, Cushman M, et al.: Cardiovascular mortality risk in chronic kidney disease: comparison of traditional and novel risk factors.
JAMA, 2005, 293: 1737-1745. [Medline] [CrossRef]

4) Sietsema KE, Amato A, Adler SG, et al.: Exercise capacity as a predictor of survival among ambulatory patients with end-stage renal disease. Kidney Int, 2004, 65: 719-724. [Medline] [CrossRef]

5) Stack AG, Molony DA, Rives T, et al.: Association of physical activity with mortality in the US dialysis population. Am J Kidney Dis, 2005, 45: 690-701. [Medline] [CrossRef]

6) Henrique DM, Reboredo MM, Chaoubah A, et al.: [Aerobic exercise improves physical capacity in patients under chronic hemodialysis]. Arq Bras Cardiol, 2010, 94: 823-828. [Medline] [CrossRef]

7) Kohzuki M, Kamimoto $\mathrm{M}$, Wu XM, et al: Renal protective effects of chronic exercise and antihypertensive therapy in hypertensive rats with chronic renal failure. J Hypertens, 2001, 19: 1877-1882. [Medline] [CrossRef]

8) Kosmadakis GC, John SG, Clapp EL, et al.: Benefits of regular walking exercise in advanced pre-dialysis chronic kidney disease. Nephrol Dial Transplant, 2012, 27: 997-1004. [Medline] [CrossRef]

9) Nationl Collaborating Centre for Chronic Conditions: Chronic Kidney Disease: National Clinical Guideline for Early Identification and Management in Adults in Primary and Secondary Care. London: Royal College of Physicians, 2008.

10) Johansen KL: Exercise and dialysis. Hemodial Int, 2008, 12: 290-300. [Medline] [CrossRef]

11) Wang C, Collet JP, Lau J: The effect of Tai Chi on health outcomes in patients with chronic conditions: a systematic review. Arch Intern Med, 2004, 164: 493-501. [Medline] [CrossRef]

12) Taylor-Piliae RE, Silva E, Sheremeta SP: Tai Chi as an adjunct physical activity for adults aged 45 years and older enrolled in phase III cardiac rehabilitation. Eur J Cardiovasc Nurs, 2012, 11: 34-43. [Medline]

13) Kim HD, Kim TY, Jae HD, et al: The effects of tai chi based exercise on dynamic postural control of parkinson's disease patients while initiating gait. J Phys Ther Sci, 2011, 23: 265-269. [CrossRef]

14) Yang X, Ko GT, So WY, et al.: Associations of hyperglycemia and insulin usage with the risk of cancer in type 2 diabetes: the Hong Kong diabetes registry. Diabetes, 2010, 59: 1254-1260. [Medline] [CrossRef]

15) Harper CR, Jacobson TA: Managing dyslipidemia in chronic kidney disease. J Am Coll Cardiol, 2008, 51: 2375-2384. [Medline] [CrossRef]

16) Tsimihodimos V, Mitrogianni Z, Elisaf M: Dyslipidemia associated with chronic kidney disease. Open Cardiovasc Med J, 2011, 5: 41-48. [Medline] [CrossRef]

17) Chen SC, Hung CC, Kuo MC, et al.: Association of dyslipidemia with rena outcomes in chronic kidney disease. PLoS ONE, 2013, 8: e55643. [Medline] [CrossRef]

18) Herzberg GR: Aerobic exercise, lipoproteins, and cardiovascular disease benefits and possible risks. Can J Appl Physiol, 2004, 29: 800-807. [Medline] [CrossRef]

19) Kelley GA, Kelley KS, Franklin B: Aerobic exercise and lipids and lipoproteins in patients with cardiovascular disease: a meta-analysis of randomized controlled trials. J Cardiopulm Rehabil, 2006, 26: 131-139, quiz 140-141, discussion 142-144. [Medline] [CrossRef]

20) Toyama K, Sugiyama S, Oka H, et al.: Exercise therapy correlates with improving renal function through modifying lipid metabolism in patients with cardiovascular disease and chronic kidney disease. J Cardiol, 2010, 56: 142-146. [Medline] [CrossRef]

21) Athyros VG, Kakafika AI, Papageorgiou AA, et al.: Statin-induced increase in HDL-C and renal function in coronary heart disease patients. Open Cardiovasc Med J, 2007, 1: 8-14. [Medline] [CrossRef]

22) Goldberg AP, Hagberg JM, Delmez JA, et al.: Exercise training improves abnormal lipid and carbohydrate metabolism in hemodialysis patients. Trans Am Soc Artif Intern Organs, 1979, 25: 431-437. [Medline] [CrossRef]

23) Park IS, Song R, Oh KO, et al.: Managing cardiovascular risks with Tai Chi in people with coronary artery disease. J Adv Nurs, 2010, 66: 282-292. [Medline] [CrossRef]

24) Lee MS, Pittler MH, Taylor-Piliae RE, et al.: Tai chi for cardiovascular disease and its risk factors: a systematic review. J Hypertens, 2007, 25 : 1974-1975. [Medline] [CrossRef]

25) Taylor-Piliae RE, Haskell WL, Stotts NA, et al: Improvement in balance, strength, and flexibility after 12 weeks of Tai chi exercise in ethnic Chinese adults with cardiovascular disease risk factors. Altern Ther Health Med, 2006, 12: 50-58. [Medline] 